\title{
EZ-ALBI Score for Predicting Hepatocellular Carcinoma Prognosis
}

\author{
Kazuya Kariyama ${ }^{a}$ Kazuhiro Nouso ${ }^{a}$ Atsushi Hiraoka ${ }^{b} \quad$ Akiko Wakuta $^{a}$

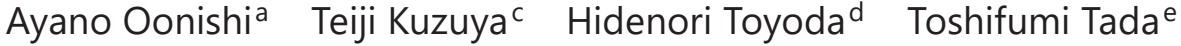 \\ Kunihiko Tsuji $^{f}$ Ei Itobayashig Toru Ishikawah ${ }^{\text {h }}$ Koichi Takaguchi $^{i}$ \\ Akemi Tsutsui $^{i}$ Noritomo Shimadaj Masatoshi Kudok ${ }^{j}$ Takashi Kumadad \\ RELPEC Study Group and HCC 48 Group \\ aDepartment of Gastroenterology and Liver Disease Center, Okayama City Hospital, \\ Okayama, Japan; ${ }^{b}$ Gastroenterology Center, Ehime Prefectural Central Hospital, \\ Ehime, Japan; 'Department of Gastroenterology and Hepatology, Nagoya University \\ Graduate School of Medicine, Nagoya, Japan; ${ }^{d}$ Department of Gastroenterology and \\ Hepatology, Ogaki Municipal Hospital, Gifu, Japan; 'Department of Gastroenterology, \\ Japanese Red Cross Society Himeji Hospital, Hyogo, Japan; ${ }^{f}$ Center of Gastroenterology, \\ Teine Keijinkai Hospital, Hokkaido, Japan; 9Department of Gastroenterology, Asahi \\ General Hospital, Chiba, Japan; hepartment of Gastroenterology, Saiseikai Niigata \\ Hospital, Niigata, Japan; 'Department of Hepatology, Kagawa Prefectural Central Hospital, \\ Kagawa, Japan; 'Department of Gastroenterology and Hepatology, Otakanomori Hospital,

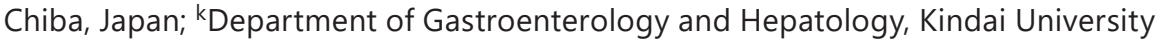 \\ Faculty of Medicine, Osaka, Japan
}

\section{Keywords}

ALBI score $\cdot$ Child-Pugh score $\cdot$ Prognostic prediction

\begin{abstract}
Introduction: The ALBI score is acknowledged as the gold standard for the assessment of liver function in patients with hepatocellular carcinoma (HCC). Unlike the Child-Pugh score, the ALBI score uses only objective parameters, albumin (Alb) and total bilirubin (T.Bil), enabling a better evaluation. However, the complex calculation of the ALBI score limits its applicability. Therefore, we developed a simplified ALBI score, based on data from a large-scale HCC database.We used the data of 5,249 naïve HCC cases registered in eight collaborating hospitals. Methods: We developed a new score, the EZ (Easy)-ALBI score, based on regression coefficients of Alb and T.Bil for survival risk in a multivariate Cox proportional hazard model. We also developed the EZ-ALBI grade and EZ-ALBI-T grade as alternative options for the ALBI grade and ALBI-T grade and evaluated their stratifying ability. Results: The equation used to calculate the EZ-ALBI score was simple $\{[T$. Bil $(\mathrm{mg} / \mathrm{dL})]-[9 \times \mathrm{Alb}(\mathrm{g} / \mathrm{dL})]\}$; this value highly cor-
\end{abstract}


related with the ALBI score (correlation coefficient, $0.981 ; p<0.0001$ ). The correlation was preserved across different Barcelona clinic liver cancer grade scores (regression coefficient, 0.93-0.98) and across different hospitals (regression coefficient, 0.98-0.99), indicating good generalizability. Although a good agreement was observed between ALBI and EZ-ALBI, discrepancies were observed in patients with poor liver function (T.Bil, $\geq 3 \mathrm{mg} / \mathrm{dL}$; regression coefficient, 0.877). The stratifying ability of EZ-ALBI grade and EZ-ALBI-T grade were good and their Akaike's information criterion values (35,897 and 34,812, respectively) were comparable with those of ALBI grade and ALBI-T grade (35,914 and 34,816, respectively). Conclusions: The EZ-ALBI score, EZ-ALBI grade, and EZ-ALBI-T grade are useful, simple scores, which might replace the conventional ALBI score in the future.

\section{Introduction}

Liver function largely reflects the prognosis of liver disease. The Child-Turcotte classification published in 1964 as a prognostic criterion for liver cirrhosis was modified by Pugh et al. [4] in 1973 and introduced as the Child-Pugh score, comprising 5 prognostic factors (prothrombin, albumin [Alb], total bilirubin [T.Bil], ascites, and encephalopathy) [1-4]. At that time, the Child-Pugh score was used exclusively to estimate short-term prognosis after treatment, such as transabdominal esophageal varicose transection or sclerotherapy. Until now, the Child-Pugh score has been used as the gold standard to assess the liver function of patients with cirrhosis. Even the prognosis of hepatocellular carcinoma (HCC) depends not only on tumor factors but also on liver function [5-8]. Especially in patients receiving surgical resection, the prognosis is significantly influenced by the background liver function [8]. In patients receiving lenvatinib, which is the most useful molecular-targeted agent currently available, patient prognosis is largely determined by the background liver function $[9,10]$. The Japan Integrated Staging (JIS) score, which combines the Child-Pugh classification with tumor factors, is usually used for long-term prognostic prediction in HCC patients [11, 12]. However, in recent years, the ALBI score using only Alb and T.Bil has attracted attention [13]. The prognostic prediction ability of ALBI-T, which combines ALBI grade and tumor factors, reportedly outperforms that of the JIS score [14-22]. In addition, the applicability of the modified ALBI (mALBI) score, which includes subdivided ALBI scores, has also been evaluated [23]. However, since the ALBI score and mALBI score require complex calculations, they have failed to prove readily applicable to clinical practice. Using a large HCC database, we here aimed to develop a simplified score based on Alb and T.Bil, the variables used in the ALBI score.

\section{Materials and Methods}

\section{Patients}

This study was based on 5,249 HCC cases registered in multiple collaborating hospitals (Asahi General Hospital, Ehime Prefectural Central Hospital, Kagawa prefectural Hospital, Ogaki Municipal Hospital, Otakanomori Hospital, Okayama City Hospital, Saiseikai Niigata Hospital, and Teine Keijinkai Hospital) from 2004 to 2018. Inclusion criterion was all HCC patients who were admitted to the hospitals and diagnosed as HCC. Exclusion criteria were (1) HCC diagnosed before January 2004, (2) cases with missing data (Fig. 1). The diagnosis of HCC was established using imaging modalities, including computed tomography, MRI, and angiography. The diagnostic criteria for HCC were based on previous reports on hyperattenuation in the arterial phase or hypoattenuation in the portal phase as determined by dynamic computed tomography or MRI with tumor staining on angiography [24]. In cases with atypical findings, the diagnosis was confirmed pathologically, using tissue obtained from a fine-needle tumor biopsy. Tumor, node, and metastasis staging for HCC 
Table 1. Characteristics of the 5,249 patients

\begin{tabular}{lc}
\hline Variable & Median (range) \\
\hline Age, yr & $71(22-98)$ \\
Gender, male, \% & $3,662(69.6)$ \\
Etiology B/C/BC/NBNC, \% & $678(12.9) / 3,083(58.6) / 25(0.5) / 1,473(28.0)$ \\
Plt, $\times 10^{4} / \mu \mathrm{L}$ & $12.6(1.2-98.0)$ \\
PT, $\%$ & $83.0(7.3-143.0)$ \\
Alb, g/dL & $3.70(1.30-7.40)$ \\
T.Bil, mg/dL & $0.80(0.10-43.40)$ \\
AFP, ng/mL & $15.20(0.30-1,454,000.0)$ \\
PIVKA-II, mAU/mL & $65.0(0.00-3,000,000.0)$ \\
Child-pugh A/B/C, \% & $3,751(71.3) / 1,249(23.7) / 259(4.9)$ \\
TNM staging I/II/III/IV, \% & $1,314(25.0) / 2,076(39.5) / 1,137(21.6) / 732(13.9)$ \\
ALBI grade $1 / 2 / 3, \%$ & $1,837(36.2) / 2,856(56.3) / 378(7.5)$ \\
JIS grade $0 / 1 / 2 / 3 / 4 / 5, \%$ & $976(19.2) / 1,780(35.1) / 1,181(23.3) / 740(14.6) / 297(5.9) / 97(1.9)$ \\
ALBI-T grade 0/1/2/3/4/5, \% & $501(9.9) / 1,564(30.8) / 1,410(27.8) / 935(18.4) / 535(10.6) / 126(2.5)$ \\
Treatment: Resection/RFA/TACE/others/BSC, \% & $1,328(25.3) / 1,969(37.4) / 1,223(23.3) / 210(4.0) / 529(10.1)$ \\
\hline
\end{tabular}

Plt, platelet; PT, prothrombin; Alb, albumin; T.Bil, total bilirubin; AFP, alfa-feto protein; PIVKA-II, protein induced by vitamin K absence or antagonist-II; TNM staging, tumor, node, and metastasis staging for HCC, was determined based on the 6th edition of "The General Rules for the Clinical and Pathological Study of Primary Liver Cancer" developed by the Liver Cancer Study Group of Japan (LCSGJ); JIS, Japan Integrated Score; RFA, radiofrequency ablation; TACE, transarterial chemoembolization; BSC, best supportive care.

Fig. 1. Flow chart of the patients. Inclusion criterion was all HCC patients who were admitted to each institution and diagnosed as HCC. Exclusion criteria were (1) HCC diagnosed before January 2004 and (2) cases with missing data. HCC, hepatocellular carcinoma; Alb, albumin; T.Bil; total bilirubin.

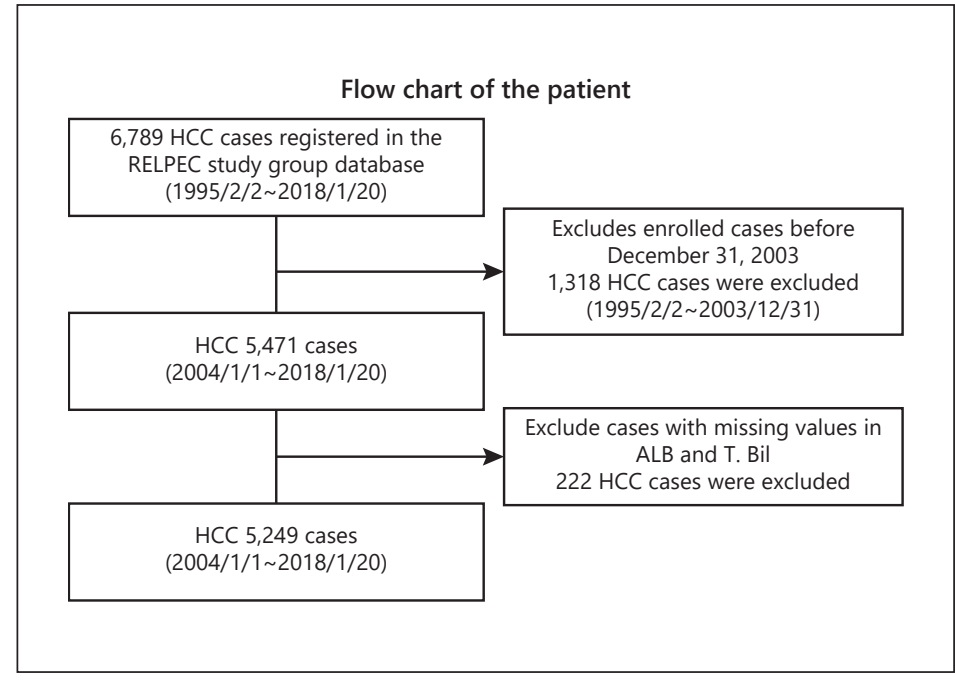

was determined based on the 6th edition of "The General Rules for the Clinical and Pathological Study of Primary Liver Cancer" developed by the Liver Cancer Study Group of Japan (LCSGJ) [25]. Albumin was measured using the modified bromocresol purple method in most of the cases.

The median age (range) at diagnosis was 71 (22-98) years, and 3,662 cases (69.6\%) were male. Over $90 \%$ of the patients were Child-Pugh A or B and ALBI-1 or ALBI-2 (Table 1). The mean observation period was 38 months.

The study protocol complied with the ethical guidelines of the World Medical Association Declaration of Helsinki and was approved by our institutional review board. The study was approved by the Ethics Committee of the IRB of Okayama City Hospital, and the approval code was 1-222, and we had approval to use our multi-institution data. 
Table 2. Risk factors for survival

\begin{tabular}{lcll}
\hline & Hazard ratio & $95 \%$ confidence interval & $p$ value \\
\hline Total bilirubin & 0.123 & $1.115-1.149$ & $<0.0001$ \\
Albumin & -0.349 & $0.321-0.375$ & $<0.0001$ \\
\hline
\end{tabular}

Development of EZ-ALBI

We performed multivariate Cox proportional hazard analysis using Alb and T.Bil values in order to calculate the hazard ratio of overall survival of HCC patients and developed the new scoring system based on the regression coefficients of Alb and T.Bil. The EZ (Easy)-ALBI score was developed by simplifying the coefficients.

Comparison of the EZ-ALBI Score with the Conventional ALBI Score

We analyzed the correlation between this newly developed EZ-ALBI score and the conventional ALBI score using regression analysis for all HCC cases. Inter-Barcelona clinic liver cancer (BCLC) stage scores and inter-hospital validation data were also analyzed.

Development and Verification of EZ-ALBI Grade and EZ-ALBI-T

We determined the cutoff values of EZ-ALBI grade $1 / 2 / 3$ that corresponded to the values of ALBI grade $1 / 2 / 3$ using the respective regression equations. Furthermore, we calculated EZ-ALBI-T grade by combining the derived EZ-ALBI grade and the clinical tumor, node, and metastasis stage. This was the same method that we used for developing ALBI-T grade [26].

Next, we analyzed the prognosis of the 5,249 HCC patients as identified by EZ-ALBI grade and compared the results with results obtained using the ALBI grade. Furthermore, we compared the prognostic ability of the EZ-ALBI-T grade with the ALBI-T grade and JIS grade using Akaike's information criterion (AIC).

\section{Statistics}

Data were expressed as median and range. All statistical analyses were performed using Easy R (Saitama Medical Center, Jichi Medical University, Saitama, Japan) [27], a graphical user interface for R (The R Foundation for Statistical Computing, Vienna, Austria) [28].

\section{Results}

EZ-ALBI Score

Hazard ratios of Alb and T.Bil for overall survival were analyzed using the multivariate Cox proportional hazard model, and the results are displayed in Table 2 . The hazards based on Alb and T.Bil were calculated using the following equation:

$$
\mathrm{h}(\mathrm{t})=\mathrm{h} 0(\mathrm{t}) \times \operatorname{EXP}((0.123 \times \mathrm{T} . \text { Bil }[\mathrm{mg} / \mathrm{dL}])-(1.053 \times \text { Alb }[\mathrm{g} / \mathrm{dL}]))
$$

We developed the new score using the core of the equation as follows:

New score $=(0.123 \times$ T.Bil $[\mathrm{mg} / \mathrm{dL}])-(1.053 \times \mathrm{Alb}[\mathrm{g} / \mathrm{dL}])$

Furthermore, we simplified the regression coefficient of this equation by using integer and created EZ-ALBI score.

EZ-ALBI score $=$ T.Bil $(\mathrm{mg} / \mathrm{dL})-(9 \times \mathrm{Alb}(\mathrm{g} / \mathrm{dL}))$ 
Fig. 2. Correlation between ALBI score and EZ-ALBI score. The correlation coefficient between ALBI and EZ-ALBI scores was 0.981 (95\% CI, 0.980-0.982). Hence, the ALBI and EZ-ALBI scores correlated very well $(p<0.0001)$. CI, confidence interval. (c) 2020 The Author(s). Published by S. Karger AG, Basel www.karger.com/lic

Kariyama et al.: EZ-ALBI Score for Hepatocellular Carcinoma Prognosis

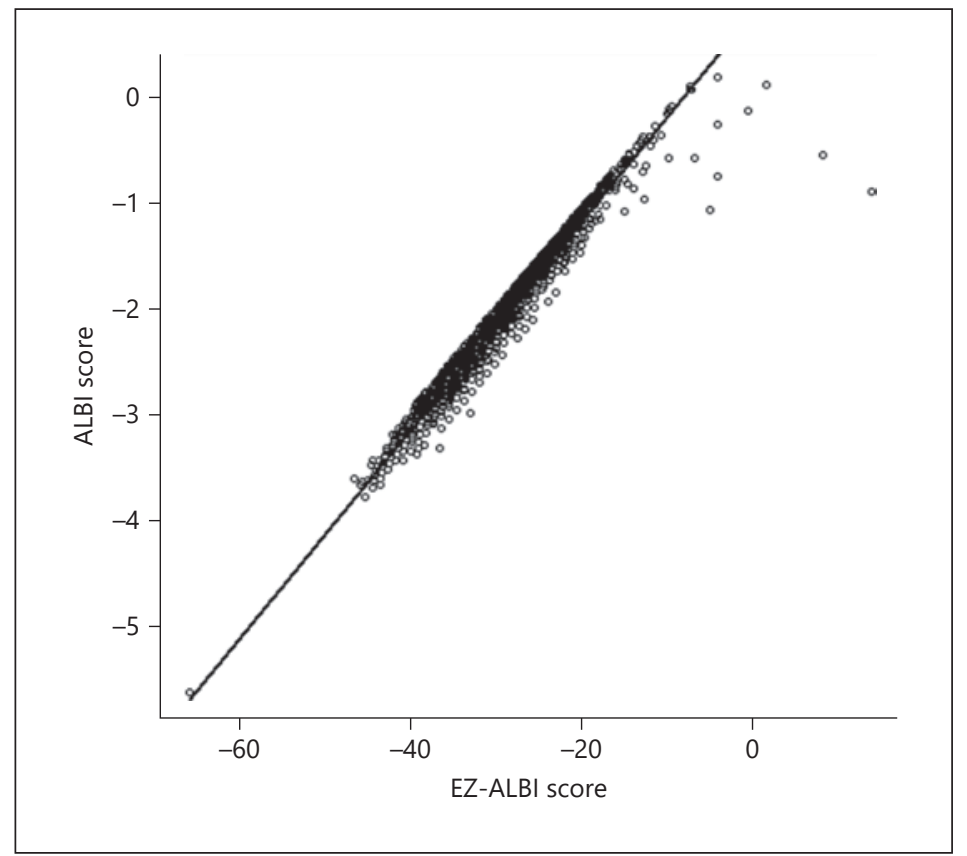

Relationship between ALBI Score and EZ-ALBI Score

The correlation between the ALBI score and EZ-ALBI score for all HCC cases is shown in Figure 2. Good linear correlation was observed, and the correlation coefficient was 0.981 (95\% confidence interval, $0.980-0.982 ; p<0.0001$ ). High correlation coefficients between ALBI and EZ-ALBI scores were observed across all BCLC grades. Hence, scores for BCLC grade $0, A, B, C$, and D were $0.984,0.983,0.982,0.971$, and 0.937 , respectively. Inter-hospital differences were examined across 9 hospitals, and their regression coefficients were 0.98 at the lowest and 0.99 at the highest. In terms of differences in liver function, the correlation coefficients for patients with high and low T.Bil levels ( $\geq 3$ and $<3 \mathrm{mg} / \mathrm{dL}$ ) were 0.877 and 0.985 , respectively.

\section{EZ-ALBI Grade}

We determined the conversion formula between EZ-ALBI score and ALBI score based on regression analysis:

$$
\begin{aligned}
& \text { ALBI }=0.099(\text { EZ-ALBI })+0.81 \\
& \text { and } \\
& \text { EZ-ALBI = } 9.75(\text { ALBI })-9.1
\end{aligned}
$$

From the cutoff value of ALBI grade and modified ALBI grade, we derived the cutoff value of EZ-ALBI grade, and the cutoffs for grade $1 / 2$ and grade $2 / 3$ were -34.4 and -22.2 , respectively. The cutoff value of the EZ-ALBI score corresponding to mALBI $2 \mathrm{a} / 2 \mathrm{~b}$ was -31.2 . Concordance rate of EZ-ALBI grade and ALBI grade was 93.9, 93.2 and 92.7\% for EZ-ALBI grade 1 , grade 2 , and grade 3 , respectively. We divided the patients into 2 groups (Ogaki Municipal hospital and Ehime prefectural Central Hospital, $N=2278$ ) and (Asahi General Hospital, Kagawa prefectural Hospital, Otakanomori Hospital, Okayama City Hospital, Saiseikai Niigata Hospital, and Teine Keijinkai Hospital, $N=2981$ ) and compared the stratification ability between EZ-ALBI grade and ALBI grade in each group. AICs were 13,173 at ALBI grade and 13,149 at EZ-ALBI grade in former group and 19,552 at ALBI grade and 19,561 at EZ-ALBI 
Kariyama et al.: EZ-ALBI Score for Hepatocellular Carcinoma Prognosis

Fig. 3 Overall survival according to ALBI grade and EZ-ALBI grade. Three- and 5-year survival rates according to ALBI grade $1 / 2 / 3$ were $80 / 58 / 18 \%$ and $67 / 40 / 8 \%$, respectively. According to EZ-ALBI grade $1 / 2 / 3$, the corresponding rates were $80 / 57 / 18 \%$ and $67 / 39 / 8 \%$, respectively. The prognostic ability of the ALBI grade and EZ-ALBI grade by AIC were 35,914 and 35,897, respectively. Both ALBI grade and EZ-ALBI grade stratified HCC patient prognosis well. AIC, Akaike's information criterion; HCC, hepatocellular carcinoma.
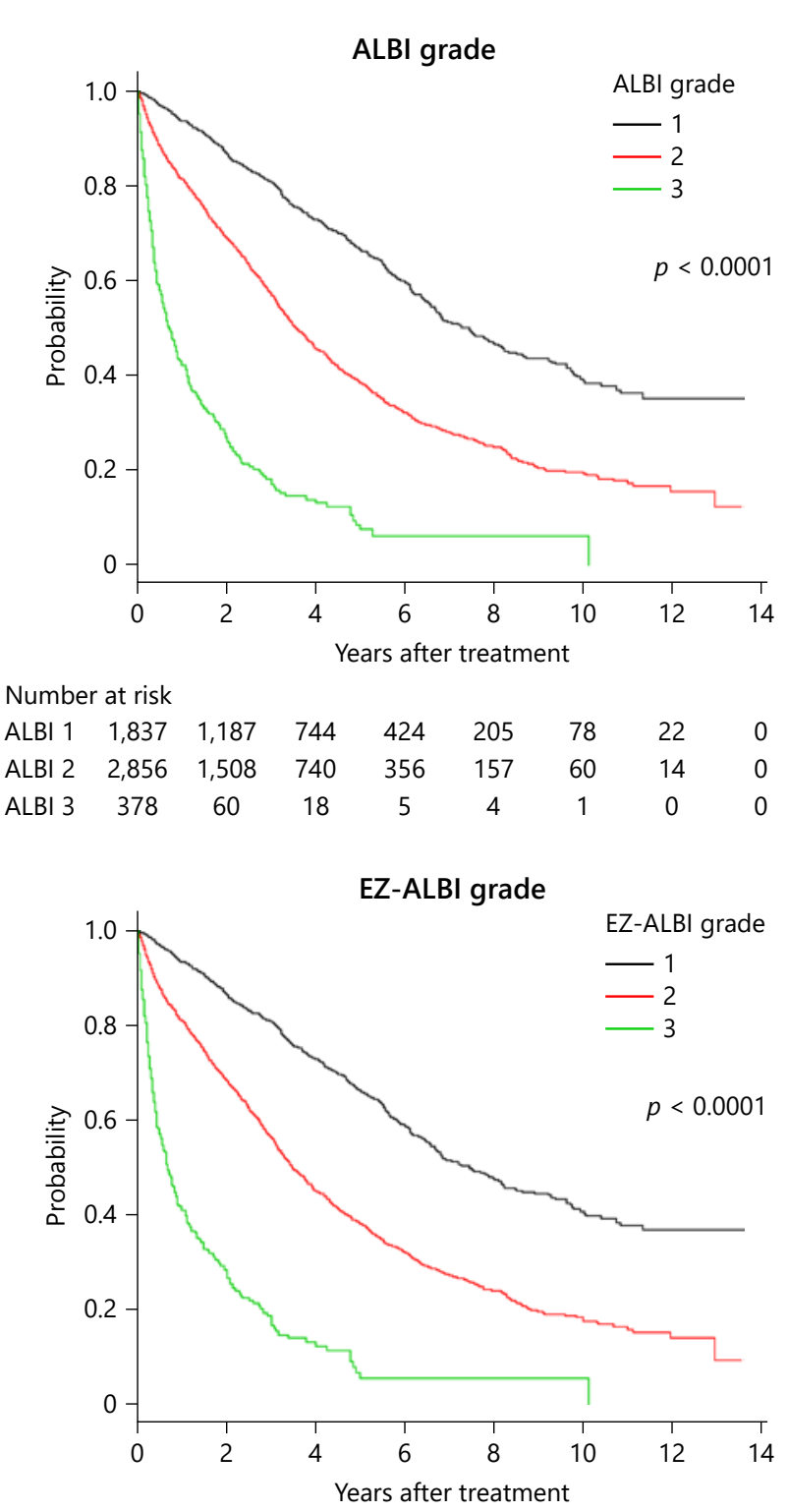

Number at risk

$\begin{array}{lllllllll}\text { EZ-ALBI } 1 & 1,836 & 1,186 & 750 & 426 & 209 & 84 & 25 & 0\end{array}$

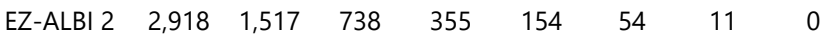

$\begin{array}{lllllllll}\text { EZ-ALBI } 3 & 314 & 52 & 14 & 4 & 3 & 1 & 0 & 0\end{array}$

grade in latter group. The difference of AIC between EZ-ALBI grade and ALBI grade was very small in both groups.

The overall survival of HCC cases by EZ-ALBI grade and ALBI grade are shown in Figure 3. Similar to the ALBI grade, the EZ-ALBI grade enabled better stratification than did the Child-Pugh classification. The stratification abilities of the ALBI grade, Child-Pugh classification, and the EZ-ALBI grade by AIC were 35,914, 37,436, and 35,897, respectively. The 3and 5-year survival rates of ALBI grade $1 / 2 / 3$ were $80 / 58 / 18 \%$ and $67 / 40 / 8 \%$, respectively, and those of the EZ-ALBI grade $1 / 2 / 3$ were $80 / 57 / 18 \%$ and $67 / 39 / 8 \%$, respectively. 
Fig. 4. Overall survival according to ALBI-T grade and EZ-ALBI-T grade. Three- and 5 -years survival rate of ALBI-T grade $0 / 1 / 2 / 3 / 4 / 5$ were $92 / 86 / 68 / 38 / 10 / 3 \%$ and $85 / 66 / 43 / 20 / 7 / 0 \%$, respectively. According to EZ-ALBI-T grade $0 / 1 / 2 / 3 / 4 / 5$, the corresponding values were $92 / 85 / 67 / 38 / 9 / 3 \%$ and $85 / 66 / 43 / 20 / 6 / 0 \%$, respectively. The stratification abilities of ALBI-T grade and EZ-ALBI-T grade by AIC were 34,816 and 34,812, respectively. AIC, Akaike's information criterion.

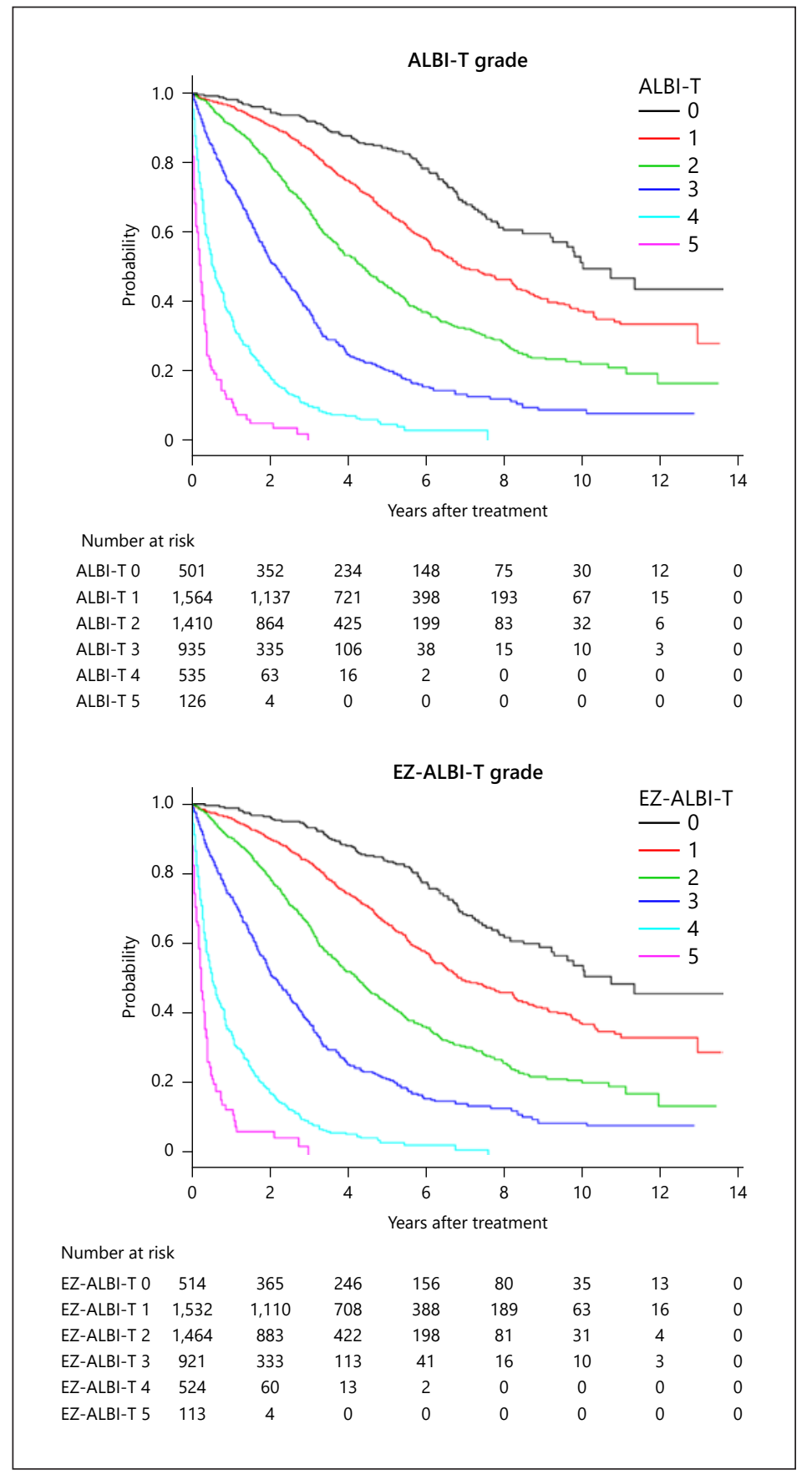

\section{EZ-ALBI-T Grade}

The overall survival of HCC patients stratified by ALBI-T grade and EZ-ALBI-T grade is shown in Figure 4. Like the ALBI-T grade, the EZ-ALBI-T grade also showed a better stratification ability than did the JIS grade. The stratification abilities of ALBI-T grade, JIS grade, and the EZ-ALBI-T grade by AIC were 34,816, 34,902, and 34,812, respectively. Three- and 5-years survival rates using ALBI-T grade 0/1/2/3/4/5 were 92/86/68/38/10/3\% and 85/66/ $43 / 20 / 7 / 0 \%$, respectively, and $92 / 85 / 67 / 38 / 9 / 3 \%$ and $85 / 66 / 43 / 20 / 6 / 0 \%$, respectively, using EZ-ALBI-T grade. 


\section{Discussion and Conclusion}

In this study, we developed a simple score, which could potentially replace the ALBI score: the EZ-ALBI score. We proved good agreement between the ALBI and EZ-ALBI scores in different situations, including different BCLC stages and different hospitals. Although minor discrepancies were observed when the T.Bil level was higher than $3 \mathrm{mg} / \mathrm{dL}$, most of these were classified into the same grade, BCLC-D, meaning that the deviation would not pose an issue in clinical use. The discrepancy of the scores in patients with high T.Bil mainly resulted from the adoption of logarithmic change of T.Bil in the ALBI score. In the EZ-ALBI Score, raw value of T.Bil was used in the formula. The EZ-ALBI score can be used for simple grading (EZ-ALBI grade) and integrated grading (EZ-ALBI-T grade), and their stratification abilities were almost equal to those of the ALBI grade and ALBI-T grade.

Unlike the ALBI score, the EZ-ALBI score is relatively easy to calculate. The ALBI score is apt for prognostic prediction of HCC and can be objectively evaluated, but the fact that it is relatively complicated to calculate has limited its dissemination and use in clinical practice. Despite the prevalence of automatic calculation software in electronic medical record systems or in some internet sites, there are still many hospitals where the systems are difficult to access. This issue can be overcome by using the EZ-ALBI score.

Simplicity is very important in algorithms and indicators used in real clinical practice, exemplified by the use of fibrosis or carcinogenic markers. Now that the electronic medical record has developed, the ALBI score can be automatically calculated by inserting the calculation formula of the ALBI score in the electronic medical record. There are also some internet sites that automatically calculate the ALBI score. However, if there is no calculation method, it is difficult to perceptually grasp liver reserve like the Child-Pugh score. The EZ-ALBI score can also be calculated by mental calculation, which makes it possible to intuitively grasp liver reserve. Although the GALAD score is based on simple factors and its prediction ability for HCC development is high, the calculation of this score is complex, and so the GALAD score has failed to diffuse into clinical practice [29].

There is a limitation in this study. We examined the utility of EZ-ALBI score only on the background of Japanese patients, and inter-country validation has not yet been performed. Since the parameters used in the EZ-ALBI score are the same as those used in the ALBI score, any difference from country to country might be small. However, an international validation study is crucial.

In this study, we demonstrated the usefulness of EZ-ALBI score, grade, and EZ-ALBI-T. They rely on objective parameters, are easy to calculate, and have the same stratification abilities as the ALBI score, grade, and ALBI-T. The EZ-ALBI series may compensate for the shortcomings of Child-Pugh score and ALBI and are expected to provide a significant contribution to clinical practice.

\section{Acknowledgements}

We gratefully acknowledge all the doctors who collaborated on this project by collecting data on the HCC patients registered in their respective hospitals.

\section{Statement of Ethics}

The study protocol conformed to the ethical guidelines of the World Medical Association Declaration of Helsinki and was approved by our institutional review board (1-222; Okayama City Hospital Institutional Review Board). We had the approval to use our multi-institution data.

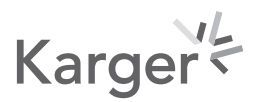




\section{Conflict of Interest Statement}

The authors have no conflicts of interest to declare.

\section{Funding Sources}

This research received no external funding.

\section{Author Contributions}

Conceptualization: T.K., M.K., A.H., and T.K.; data curation: H.T., T.T., A.H., K.T., E.I., T.I., A.T., N.S., A.W., A.O., and T.K.; methodology: T.K.; writing - original draft: K.K.; writing - review and editing: K.N.

\section{References}

1 Child CG, Turcotte JG. Surgery and portal hypertension. In: Child CG, editor. The liver and portal hypertension. Philadelphia: WB Saunders Co.; 1964. Vol. 1; p. 1.

2 Wantz GE, Payne MA. Experience with portacaval shunt for portal hypertension. N Engl J Med. 1961 Oct;265: 721-8.

3 Turcotte JG, Lambert MJ. Variceal hemorrhage, hepatic cirrhosis, and portacaval shunts. Surgery. 1973 Jun; 73(6):810-7.

4 Pugh RN, Murray-Lyon IM, Dawson JL, Pietroni MC, Williams R. Transection of the oesophagus for bleeding oesophageal varices. Br J Surg. 1973 Aug;60(8):646-9.

5 Llovet JM. Updated treatment approach to hepatocellular carcinoma. J Gastroenterol. 2005 Mar;40(3):22535 .

6 Huitzil-Melendez FD, Capanu M, O’Reilly EM, Duffy A, Gansukh B, Saltz LL, et al. Advanced hepatocellular carcinoma: which staging systems best predict prognosis? J Clin Oncol. 2010 Jun 10;28(17):2889-95.

7 Kudo M, Trevisani F, Abou-Alfa GK, Rimassa L. Hepatocellular carcinoma: therapeutic guidelines and medical treatment. Liver Cancer. 2016 Nov;6(1):16-26.

8 Toyoda H, Lai PB, O'Beirne J, Chong CC, Berhane S, Reeves H, et al. Long-term impact of liver function on curative therapy for hepatocellular carcinoma: application of the ALBI grade. Br J Cancer. 2016 Mar; 114(7): $744-50$.

9 Hiraoka A, Kumada T, Atsukawa M, Hirooka M, Tsuji K, Ishikawa T, et al. Important clinical factors in sequential therapy including lenvatinib against unresectable hepatocellular carcinoma. Oncology. 2019;97(5):277-85.

10 Hiraoka A, Kumada T, Atsukawa M, Hirooka M, Tsuji K, Ishikawa T, et al. Prognostic factor of lenvatinib for unresectable hepatocellular carcinoma in real-world conditions-multicenter analysis. Cancer Med. 2019 Jul; 8(8):3719-28.

11 Kudo M, Chung H, Osaki Y. Prognostic staging system for hepatocellular carcinoma (CLIP score): its value and limitations, and a proposal for a new staging system, the Japan Integrated Staging Score (JIS score). J Gastroenterol. 2003 Mar;38(3):207-15.

12 Kudo M, Chung H, Haji S, Osaki Y, Oka H, Seki T, et al. Validation of a new prognostic staging system for hepatocellular carcinoma: the JIS score compared with the CLIP score. Hepatology. 2004 Dec;40(6):1396-405.

13 Johnson PJ, Berhane S, Kagebayashi C, Satomura S, Teng M, Reeves HL, et al. Assessment of liver function in patients with hepatocellular carcinoma: a new evidence-based approach-the ALBI grade. J Clin Oncol. 2015 Feb;33(6):550-8.

14 Hiraoka A, Kumada T, Michitaka K, Toyoda H, Tada T, Ueki H, et al. Usefulness of albumin-bilirubin grade for evaluation of prognosis of 2584 Japanese patients with hepatocellular carcinoma. J Gastroenterol Hepatol. 2016 May;31(5):1031-6.

15 Hiraoka A, Kumada T, Michitaka K, Kudo M. Newly proposed ALBI grade and ALBI-T score as tools for assessment of hepatic function and prognosis in hepatocellular carcinoma patients. Liver Cancer. 2019 Oct: $8(5): 312-25$.

16 Wang YY, Zhong JH, Su ZY, Huang JF, Lu SD, Xiang BD, et al. Albumin-bilirubin versus child-pugh score as a predictor of outcome after liver resection for hepatocellular carcinoma. Br J Surg. 2016 May;103(6):725-34.

17 Hiraoka A, Kumada T, Kudo M, Hirooka M, Tsuji K, Itobayashi E, et al. Albumin-bilirubin (ALBI) grade as part of the evidence-based clinical practice guideline for HCC of the Japan society of hepatology: a comparison with the liver damage and child-pugh classifications. Liver Cancer. 2017 Jun;6(3):204-15.

18 Hiraoka A, Kumada T, Hirooka M, Tsuji K, Itobayashi E, Kariyama K, et al. A better method for assessment of hepatic function in hepatocellular carcinoma patients treated with radiofrequency ablation: usefulness of albumin-bilirubin grade. Hepatol Res. 2018 Feb;48(3):E61-7. 
Kariyama et al.: EZ-ALBI Score for Hepatocellular Carcinoma Prognosis

19 Hiraoka A, Kumada T, Kudo M, Hirooka M, Koizumi Y, Hiasa Y, et al. Hepatic function during repeated TACE procedures and prognosis after introducing Sorafenib in patients with unresectable hepatocellular carcinoma: multicenter analysis. Dig Dis. 2017;35(6):602-10.

20 Hiraoka A, Michitaka K, Kumada T, Izumi N, Kadoya M, Kokudo N, et al. Validation and potential of albuminbilirubin grade and prognostication in a nationwide survey of 46,681 hepatocellular carcinoma patients in Japan: the need for a more detailed evaluation of hepatic function. Liver Cancer. 2017 Nov;6(4):325-36.

21 Izumoto H, Hiraoka A, Ishimaru Y, Murakami T, Kitahata S, Ueki H, et al. Validation of newly proposed time to transarterial chemoembolization progression in intermediate-stage hepatocellular carcinoma cases. Oncology. 2017;93(Suppl 1):120-6.

22 Tada T, Kumada T, Toyoda H, Tsuji K, Hiraoka A, Michitaka K, et al. Impact of albumin-bilirubin grade on survival in patients with hepatocellular carcinoma who received sorafenib: an analysis using time-dependent receiver operating characteristic. J Gastroenterol Hepatol. 2019 Jun;34(6):1066-73.

23 Hiraoka A, Kumada T, Tsuji K, Takaguchi K, Itobayashi E, Kariyama K, et al. Validation of modified ALBI grade for more detailed assessment of hepatic function in hepatocellular carcinoma patients: a multicenter analysis. Liver Cancer. 2019 Mar;8(2):121-9.

24 Kudo M, Kitano M, Sakurai T, Nishida N. General rules for the clinical and pathological study of primary liver cancer, nationwide follow-up survey and clinical practice guidelines: the outstanding achievements of the liver cancer study group of Japan. Dig Dis. 2015 Oct;33(6):765-70.

25 Liver Cancer Study Group of Japan. The general rules for the clinical and pathological study of primary liver cancer. 6th ed. Tokyo, Japan: Kanehara \& Co.; 2015. p. 26-30.

26 Hiraoka A, Kumada T, Michitaka K, Toyoda H, Tada T, Ueki H, et al. Usefulness of albumin-bilirubin grade for evaluation of prognosis of 2584 Japanese patients with hepatocellular carcinoma. J Gastroenterol Hepatol. 2016 May;31(5):1031-6.

27 Kanda Y. Investigation of the freely available easy-to-use software 'EZR' for medical statistics. Bone Marrow Transplant. 2013 Mar;48(3):452-8.

28 R Development Core Team RFfSC. R: a language and environment for statistical computing. Vienna, Austria: The R Foundation for Statistical Computing; 2005.

29 Johnson PJ, Pirrie SJ, Cox TF, Berhane S, Teng M, Palmer D, et al. The detection of hepatocellular carcinoma using a prospectively developed and validated model based on serological biomarkers. Cancer Epidemiol Biomarkers Prev. 2014;23(1):144-53. 